\title{
Resonant activation in piecewise linear asymmetric potentials
}

\author{
Alessandro Fiasconaro ${ }^{1,2, *}$ and Bernardo Spagnolo ${ }^{3, \dagger}$ \\ ${ }^{1}$ Centro Universitario de la Defensa de Zaragoza, Ctra. de Huesca s/n, E-50090 Zaragoza, Spain \\ ${ }^{2}$ Departamento de Física de la Materia Condensada and ICMA (CSIC-Universidad de Zaragoza), E-50009 Zaragoza, Spain \\ ${ }^{3}$ Dipartimento di Fisica, Group of Interdisciplinary Physics, Università di Palermo and CNISM, Viale delle Scienze, I-90128 Palermo, Italy
}

(Received 13 June 2010; published 22 April 2011)

\begin{abstract}
This work analyzes numerically the role played by the asymmetry of a piecewise linear potential, in the presence of both a Gaussian white noise and a dichotomous noise, on the resonant activation phenomenon. The features of the asymmetry of the potential barrier arise by investigating the stochastic transitions far behind the potential maximum, from the initial well to the bottom of the adjacent potential well. Because of the asymmetry of the potential profile together with the random external force uniform in space, we find, for the different asymmetries: (1) an inversion of the curves of the mean first passage time in the resonant region of the correlation time $\tau$ of the dichotomous noise, for low thermal noise intensities; (2) a maximum of the mean velocity of the Brownian particle as a function of $\tau$; and (3) an inversion of the curves of the mean velocity and a very weak current reversal in the miniratchet system obtained with the asymmetrical potential profiles investigated. An inversion of the mean first passage time curves is also observed by varying the amplitude of the dichotomous noise, behavior confirmed by recent experiments.
\end{abstract}

DOI: 10.1103/PhysRevE.83.041122

PACS number(s): 05.40.-a, 05.45.-a, 02.50.Ey

\section{INTRODUCTION}

Resonant activation (RA) is one of the well-studied noiseinduced phenomena for thermally activated barrier crossing problems. For a Brownian particle surmounting a fluctuating potential barrier from an initial metastable state, the signature of the RA effect is the presence of a minimum of the mean escape time as a function of the mean switching frequency of the external force. The RA phenomenon is important for investigating the transient dynamics in physical, chemical, and biological systems [1-22]. Very recently, a strategy against bacterial persistence based on resonant activation was proposed in Ref. [23]. Moreover, RA was investigated in a system with a piecewise linear potential and a multistate noise [24], in a thermal-inertial ratchet [25], and was experimentally observed in single-electron escape from a metastable state over an oscillating barrier in a silicon-based ratchet transfer [26]. Specifically, the role of a constant bias force on the RA phenomenon was investigated in Ref. [25]; in Ref. [26], an inversion of the curve of the mean first passage time (MFPT) in the resonant region was experimentally observed, with differences in the MFPT between the lowerand resonance-frequency regimes, by increasing the amplitude of the modulating radio frequency (rf) signal.

The RA phenomenon was theoretically investigated in Refs. [1-16,23-25] and experimentally revealed in Refs. [17-22,26]. The occurrence of the RA together with other stochastic effects such as noise-enhanced stability [12,20,27-29] and stochastic resonance [30] have been also investigated [11-14,19,20]. The RA effect is characterized by the strong correlation between the mean time of the potential fluctuations and the crossing time over the barrier, and it is totally different from the dynamic resonance requiring the matching between the driving frequency and the natural

\footnotetext{
*afiascon@unizar.es

${ }^{\dagger}$ http://gip.dft.unipa.it
}

frequency of the system [31]. In the seminal paper by Doering and Gadua [1], a switching piecewise linear potential in a range $[0, L]$ with fixed minima in $x=0$ and $x=L$ was considered. A slightly different choice was made by Bier and Astumiam [2], who used a piecewise linear potential with the height of the barrier fluctuating between $V_{0}-b$ and $V_{0}+b$, with $V_{0}$ the height of the average barrier and $b<V_{0}$, thus maintaining the presence of the barrier in all the dynamics.

Both choices have in common that the potential can be considered as symmetrical in shape and maintains the same value at the two extremes in all dynamics $[V(0)=V(L)]$.

The dependence of the RA effect on the shape of potentials has been previously investigated in Refs. $[4,5,7,10]$, but there is lack of studies on the role played by the asymmetry of the potential barrier in this phenomenon. In a very recent paper [16], the thermally activated crossing of an asymmetric fluctuating barrier was investigated, by using two different scalings of the dichotomous noise.

The aim of this work, which is a more refined analysis of a previous preliminary investigation [15] (see also Ref. [47] in Ref. [16]), is to focus on the role played by the asymmetry of a piecewise linear potential in the RA effect. We solve numerically the Langevin equation associated with this potential and calculate the MFPT and the mean velocity of the Brownian particle crossing the potential barrier. The asymmetry of the potential barrier has been investigated by means of the stochastic transitions far behind the potential maximum, from the initial well to the bottom of the adjacent potential well. Owing to the asymmetry of the fluctuating potential profile together with the randomly fluctuating external force uniform in space, we find the following: (1) an inversion of the MFPT curves, related to different asymmetric potentials, as a function of the correlation time $\tau$ of the dichotomous noise, for low thermal noise intensities, (2) a maximum of the mean velocity of the Brownian particle as a function of $\tau$, and (3) a weak current reversal in the miniratchet system obtained with the asymmetrical potential profiles investigated. We also find a reversal behavior for the MFPT with different 
amplitude of the dichotomous noise. The same behavior has been observed experimentally in Ref. [26] [see their Fig. 5(a)]. Some comparisons with smooth-shaped symmetric potentials have been also performed.

\section{MODEL AND RESULTS}

The overdamped dynamics of the Brownian particle crossing the fluctuating potential barrier is described by the following Langevin equation:

$$
\dot{x}=-V^{\prime}(x)+\eta(t)+\xi(t)=-U^{\prime}(x, t)+\xi(t),
$$

with

$$
U(x, t)=V(x)-x \eta(t)
$$

and

$$
V(x)= \begin{cases}\frac{h x}{x_{m}} & x \leqslant x_{m} \\ \frac{h(L-x)}{L-x_{m}} & x \geqslant x_{m},\end{cases}
$$

where $\xi(t)$ is the Gaussian white noise, with zero mean and correlation function $\left\langle\xi(t) \xi\left(t^{\prime}\right)\right\rangle=2 D \delta\left(t-t^{\prime}\right)$, and $D=k_{B} T$ is the noise intensity, with $T$ the absolute temperature and $k_{B}$ the Boltzmann constant. In Eq. (1) the time is measured in terms of the damping parameter $\gamma$ with the scaling $t=t_{R} / \gamma$, with $t_{R}$ the real time. The random force $\eta(t)$ is a dichotomous noise source, which takes here the two values $\{-a, a\}$ with an exponential correlation function $\left\langle\eta(t) \eta\left(t^{\prime}\right)\right\rangle=(Q / \tau) e^{-\left|t-t^{\prime}\right| / \tau}$, with $Q=a^{2} \tau$ and correlation time $\tau$. The potential $U(x, t)$ fluctuates randomly between two configurations, $V_{+}(x)$ (up) and $V_{-}(x)$ (down), remaining in one of the two states for the average time $\tau$ before switching to the other. We have

$$
V_{ \pm}(x)=V(x) \pm a x .
$$

In Eq. (3), the values of parameters are $L=1, h=2$, and $x_{m}=L / 2+k$. Here $k$ represents the asymmetry parameter, defined as the distance between the position of the maximum of the potential $x_{m}$ and the position of the symmetrical maximum $x_{s}$ (here $\left.x_{s}=0.5\right)$.

In Fig. 1 we report the "up" and "down" configurations of the switching piecewise linear potential used in the calculations. Specifically we have in panel (a) a symmetric piecewise linear potential, the same as that used in Refs [1,2], with $V_{ \pm}(0)=V_{ \pm}(L)$; in panel (b) a symmetric piecewise linear potential with the randomly fluctuating external force uniform in space and $V_{ \pm}(0) \neq V_{ \pm}(L)$, obtained from Eqs. (3) and (4) with $k=0$; and in panel (c) an asymmetric piecewise linear potential with the randomly fluctuating external force uniform in space, obtained from Eqs. (3) and (4) with $k=$ -0.25 . In panel (c) we report also the average asymmetric piecewise linear potential. The amplitude of the random force is $a=1.2$.

The presence of the asymmetry together with the fluctuating external force uniform in space as shown in panels (b) and (c) of Fig. 1 has peculiar consequences for the behavior of the RA phenomenon.

The potential here defined by Eqs. (2) and (3) can be considered as a base modulus of the piecewise linear ratchet subjected to a dichotomous noise source, widely used in the literature [32-34].

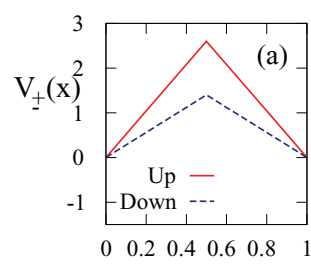

$\mathrm{X}$

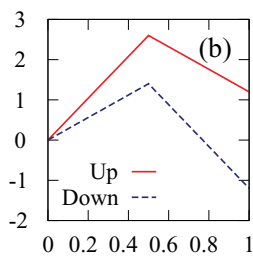

$\mathrm{x}$

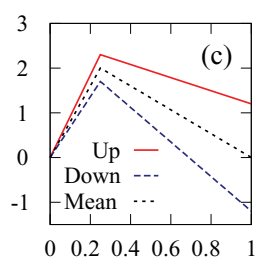

FIG. 1. (Color online) "Up" and "down" configurations of the switching piecewise linear potential $V_{ \pm}(x)$ used in our calculations. The position $x_{0}=0$ represents the starting point of the simulations. A reflecting boundary is assumed at $x=0$ and an absorbing boundary at $x=L$. (a) Symmetric piecewise linear potential the same as that used in Refs. [1,2] with $V_{ \pm}(0)=V_{ \pm}(L)$; (b) symmetric piecewise linear potential with the randomly fluctuating external force uniform in space and $V_{ \pm}(0) \neq V_{ \pm}(L)$, obtained from Eqs. (3) and (4) with $k=0$; (c) asymmetric piecewise linear potential with the randomly fluctuating external force uniform in space, obtained from Eqs. (3) and (4) with $k=-0.25$. In panel (c) we report also the average asymmetric piecewise linear potential. The values of the other parameters are $L=1, h=2$ and $x_{m}=L / 2+k, x_{s}=0.5$, and $a=1.2$.

Equation (1) has been solved numerically by using a time step $\Delta t=10^{-3}$, and the averages have been performed over a sampling of at least $N=50000$ realizations. In the $i$ th realization the particle is put at the starting position $x_{0}=0$, and the time $t_{i}$ to cross the position $x=L$ is computed. A reflecting boundary is assumed at $x=0$, in the left extremum of the potential, and an absorbing boundary at the right extremum $x=L$, after the particle surmounts the potential barrier. The ensemble average of the $t_{i}$ gives the MFPT, which presents the evidence of the RA effect, i.e., a wellpronounced minimum as a function of the correlation time $\tau$, for all the cases here studied. The results of our calculations for asymmetrical and symmetrical potentials with random external force uniform in space, that is, with $V_{ \pm}(0) \neq V_{ \pm}(L)$, are shown in Fig. 2. We notice that for the three values of the asymmetry parameter $k$, we find the resonant correlation time around the same interval $\tau_{R} \sim 10$, but with different values of the corresponding resonant MFPTs (here called $T_{R}$ ). These decrease by increasing the asymmetry parameter $k$.

We notice that the resonant region shows an inversion of the behavior of the MFPT curves for the three potential profiles investigated, with respect to both the low and high mean correlation times. Indeed, for $\tau$ lower than $\tau_{C_{L}} \approx 10^{-1}$ the curves show a higher MFPT for positive asymmetry $(k=0.25)$ and lower for negative asymmetry $(k=-0.25)$, and the same qualitative behavior is present for long mean correlation times ( $\tau$ higher than $\tau_{C_{R}} \approx 10^{3}$ ). In the intermediate region $\tau \in\left[\tau_{C_{L}}, \tau_{C_{R}}\right]$ ( $\mathrm{L}$ and $\mathrm{R}$ mean left and right, respectively), where we also find the resonant values $T_{R}$, the situation is inverted: the highest $T_{R}$ value corresponds to the negative asymmetry parameter and the lowest $T_{R}$ to the positive one. By increasing the absolute value of the asymmetry parameter $k$, the corresponding curves show, more pronounced, the same behavior here presented.

On the other hand, calculations performed with the same model of Refs. [1,2], that is, by using fixed extremes in asymmetric piecewise linear potentials and the same potential 


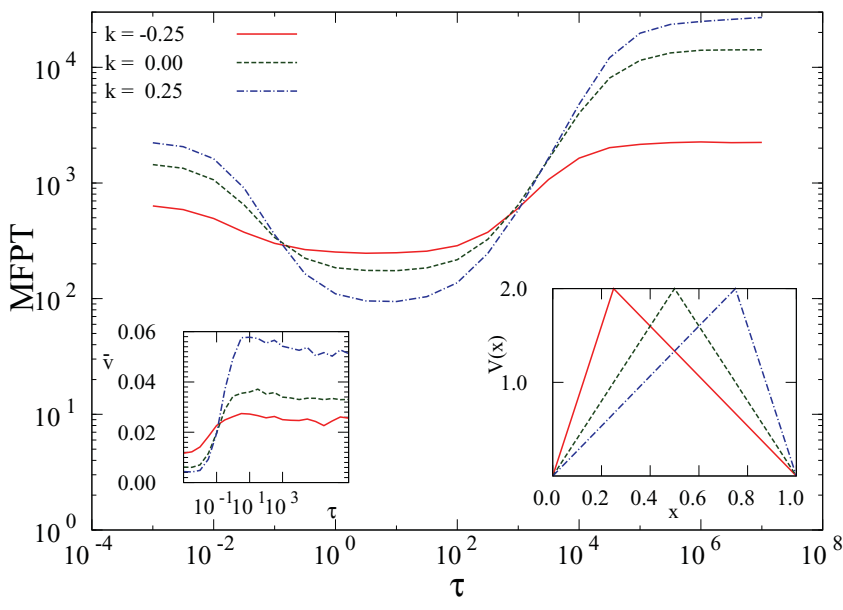

FIG. 2. (Color online) Log-log plot of MFPT as a function of the correlation time of the dichotomous noise. The RA phenomenon is observed for three values of the asymmetry parameter $k$ of the piecewise linear potential $[k=-0.25, k=0$ (i.e., symmetric potential), $k=0.25$ ], with randomly fluctuating external force uniform in the space. The value of the white noise intensity is $D=0.18$. The amplitude of the dichotomous force is $a=1.2$. The left inset shows, in a semilog plot, the mean velocity of the Brownian particle with the same asymmetries as a function of the correlation time $\tau$. The inset on the right shows the three static (average) potential profiles investigated. (The legend is the same for all the plots.) All the other parameter values are as in Fig. 1.

heights $(a=0.6)$ for all the asymmetries, give very different curves. In fact, as we can see from Fig. 3, no crossings are present between the MFPT curves for the asymmetries considered.

The inversion of the behavior of the MFPT curves, and consequently the presence of the two intersections,

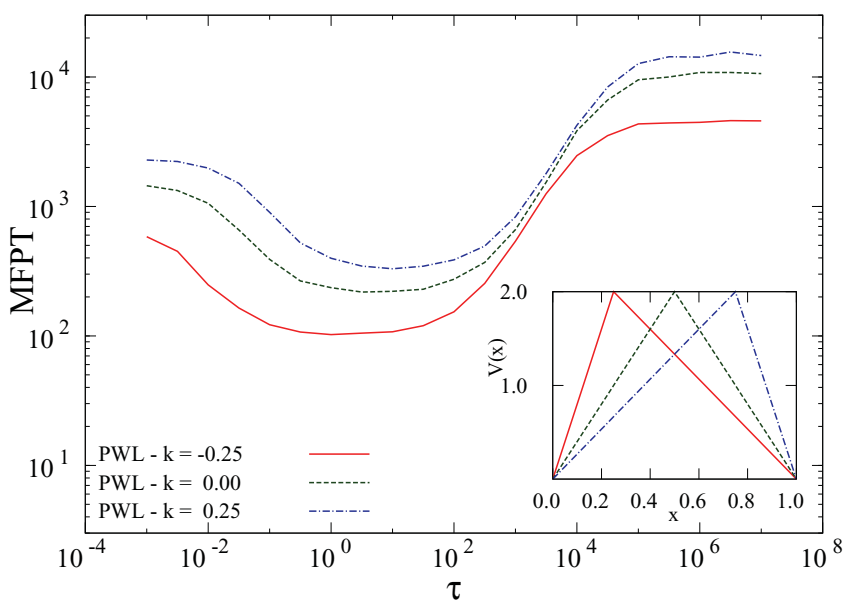

FIG. 3. (Color online) Log-log plot of MFPT as a function of the correlation time. The RA effect is observed for different asymmetrical piecewise linear (PWL) potentials with fixed extremes and equal potential heights in the three cases. Differently from the results in Fig. 2 we do not find any intersection between the three curves, and the resonant correlation time $\tau_{R}$ changes for the different asymmetries. The amplitude of the dichotomous force is $a=0.6$, the same as that corresponding to the symmetric case in Fig. 2. All the other parameter values are as in Fig. 1. approximatively at $\tau_{C_{L}}$ and $\tau_{C_{R}}$, is uniquely present in the MFPTs calculated for asymmetric potentials, by using a fluctuating force uniform over all the range $[0, L]$. This inversion does not appear either by using symmetric potentials having different shapes (see Fig. 8) or by using the asymmetric ones with fixed extremes and equal potential barrier heights (Fig. 3). In other words, the comparison between the results plotted in Figs. 2, 3, and 8 highlights that the crossing feature of the MFPT curves occurs not merely because of the asymmetry of the potential profiles, but, instead, because of the presence of the asymmetry together with the randomly fluctuating external force uniform in space $\eta(t)$.

These features explain why we find higher MFPTs for positive asymmetry than those obtained with negative asymmetry, for both low and high values of correlation time $\tau$. In fact, for very low correlation time, i.e., for very fast fluctuations of the potential profile, the Brownian particle "sees" the average barrier [1] (see inset on the right of Fig. 2). Because of the different slopes of the potential, the particle will spend more time in the interval $x<x_{m}$ for potential profile with positive asymmetry than in the case of negative asymmetry. As a consequence the MFPTs to reach the boundary at $x=1$ for positive asymmetry will be higher than those for negative asymmetry (see the references in Ref. [28] on noise-enhanced stability for the role of the slopes of the potential profile on the MFPT). For very high correlation time, that is, for very slow fluctuations of the potential barrier, the MFPT is equal to the average of the crossing times over upper and lower configurations of the barrier, and the slowest process determines the value of the average escape time [1]. In that case we have the same role of the different slopes, and the MFPTs for positive asymmetry will be higher than those obtained with the negative one.

Concerning the resonant region of $\tau$, the inversion of the MFPT curves for the various asymmetries is due to the different barrier heights of the fluctuating potential. In fact, the resonant MFPT values $T_{R}$ are determined mainly by the mean escape time over the lower of the two barriers $\left(V_{-}\right)$[8], that is,

$$
T_{R} \sim\left(1 / V_{-}^{2}\right) e^{V_{-} / D}
$$

According to that, by increasing the value of $k$, the MFPT at resonance becomes lower and lower. That is why we find, in the resonant range of values of $\tau$ and at low noise intensities, that $T_{R}$ values are lower for the positive asymmetry case than for the negative one.

The model here investigated presents interesting features in the MFPT: First, it has a value of the resonant correlation time $\tau_{R}$ not too strongly dependent on the asymmetry parameter $k$; second, it presents two correlation time intervals, close to $\tau_{C_{L}} \approx 10^{-1}$ and close to $\tau_{C_{R}} \approx 10^{3}$, with approximatively the same MFPT for all the $k$ parameters. Moreover, the crossing features of the MFPT behavior as a function of the correlation times do not occur for any value of the thermal noise. A set of calculations to check the crossing of the MFPT curves has been performed, and the related results are shown in Fig. 4, where the typical behavior of the RA effect is shown for different values of the noise intensity, namely, $D=0.2,0.25,0.4,0.6$. At low noise intensities $D$, the crossings between the curves are maintained up to the threshold value $D_{T}$. For higher noise 

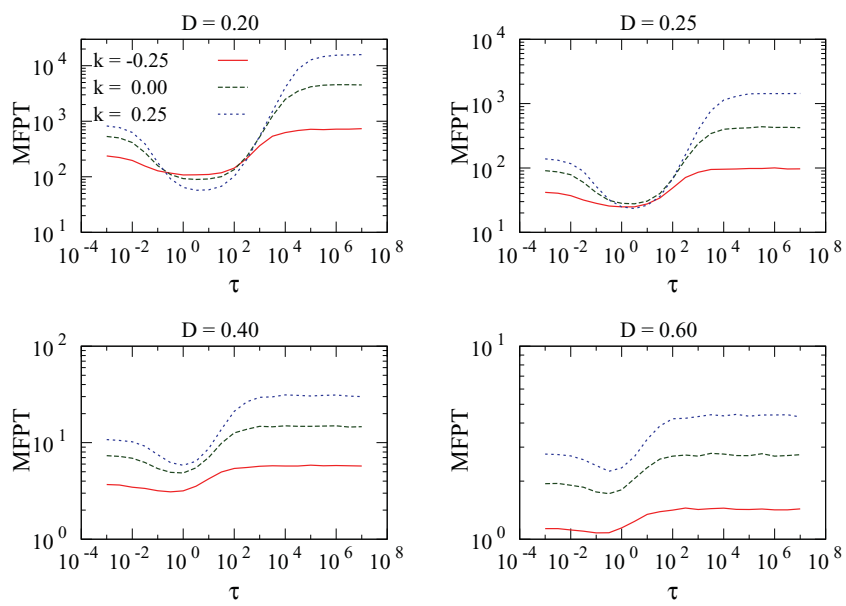

FIG. 4. (Color online) Log-log plot of MFPT as a function of the correlation time for different values of the noise intensity $D$ and asymmetry parameter $k: D=0.20,0.25,0.40,0.60$. The values of $k$ and all the other parameters are the same as in Fig. 2. We observe the RA effect for all the noise intensities investigated, the disappearance of the crossings in the MFPT curves, and the shift of the minima by increasing the noise intensity $D$.

intensities, no intersection appear in the curves. A shift of the resonant mean switching time is observed together with lower values of the related values of $T_{R}$. This is in agreement with the expression of Eq. (5) of the resonant MFPT and with the physical picture of the RA phenomenon $[1,2,8]$; that is, at an intermediate range of correlation time, the crossing event is strongly correlated with the potential fluctuations and the MFPT exhibits a minimum. By increasing the noise intensity, the $T_{R}$ decreases and the resonant correlation time as well.

The increase of the noise intensity has the effect of speeding up the escape process from the left potential well. These behaviors are shown in Fig. 5, where the resonant MFPT values $T_{R}$ are plotted as a function of the noise intensity $D$, for the three values of the asymmetry parameter $k$.

We can see that going beyond the threshold noise value $D_{T}$, the three $T_{R}$ curves invert their relative position. This noise threshold corresponds to the presence (for $D<D_{T}$ ) or the absence (for $D>D_{T}$ ) of the two crossings of the MFPT curves visible in Figs. 2 and 4.

We notice that $D_{T}$ is not unique for all the asymmetries investigated. We calculated the dependence of $\tau_{C_{L}}$ and $\tau_{C_{R}}$ on the noise intensity $D$ and on the fluctuation amplitude $a$, and that of the threshold $D_{T}$ on the amplitude $a$. The results are shown in Fig. 6. We can see that $D_{T}$ decreases with decreasing the amplitude $a$ because the difference of the potential barrier heights decreases. Of course, when $a \rightarrow 0$, the escape process is essentially to cross the barriers of fixed potential profiles, so no frequency dependence is expected for the activation phenomenon. In this case the values of MFPT for the different asymmetry parameter $k$ are the same as in the Figs. 3 and 4 in the limit of small $\tau$.

The right bottom panel of Fig. 6 shows the MFPT as a function of the correlation time $\tau$ for different values of the amplitude $a$ of the fluctuating force. We notice there another peculiar region of the activation process: a crossing of the curves at $\tau_{a} \approx 70$ with the MFPT having the same

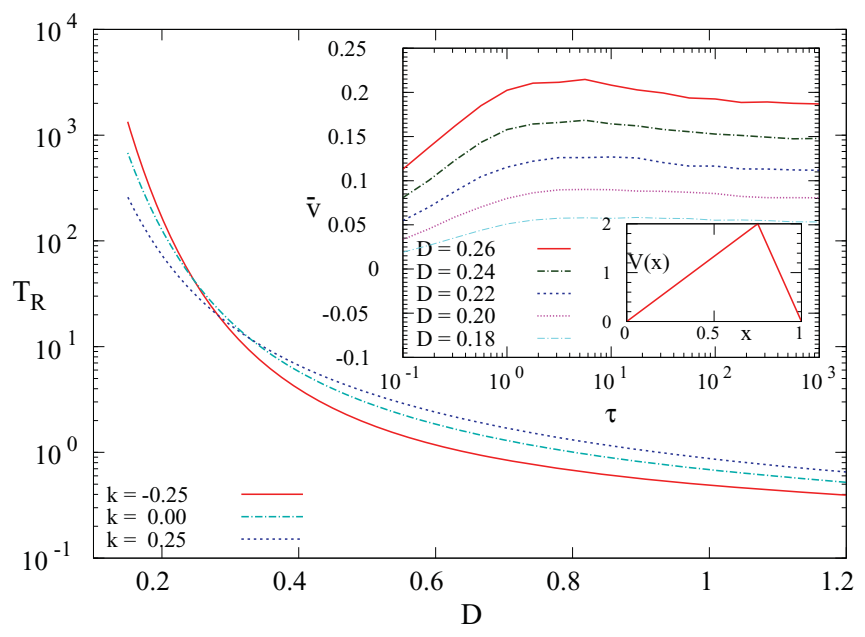

FIG. 5. (Color online) Semilog plot of the resonant MFPT values $\left(T_{R}\right)$ as a function of the noise intensity $D$ for the three asymmetries investigated. The value $D_{T} \approx 0.27$ represents the threshold of thermal noise that discriminates if the two crossings in MFPT curves of Fig. 2 are present $\left(D<D_{T}\right)$ or absent $\left(D>D_{T}\right)$. Inset: mean velocity of the Brownian particle for one value of the asymmetry parameter, $k=$ 0.25 , as a function of the correlation time $\tau$ for the noise intensities $D=0.18,0.20,0.22,0.24,0.26$. All the other parameter values are as in Fig. 1.

value obtained at the high-frequency limit. At that limit, the Brownian particle "sees" the same average potential profile, independently of the amplitude $a$. This peculiar behavior has been observed experimentally in the resonant escape over an oscillating barrier in a single-electron ratchet transfer [26] [see their Fig. 5(a)]. Specifically, in that paper, the dichotomously oscillating barrier is obtained by modulating the constant voltage applied to the center gate of the Si nanowire with a
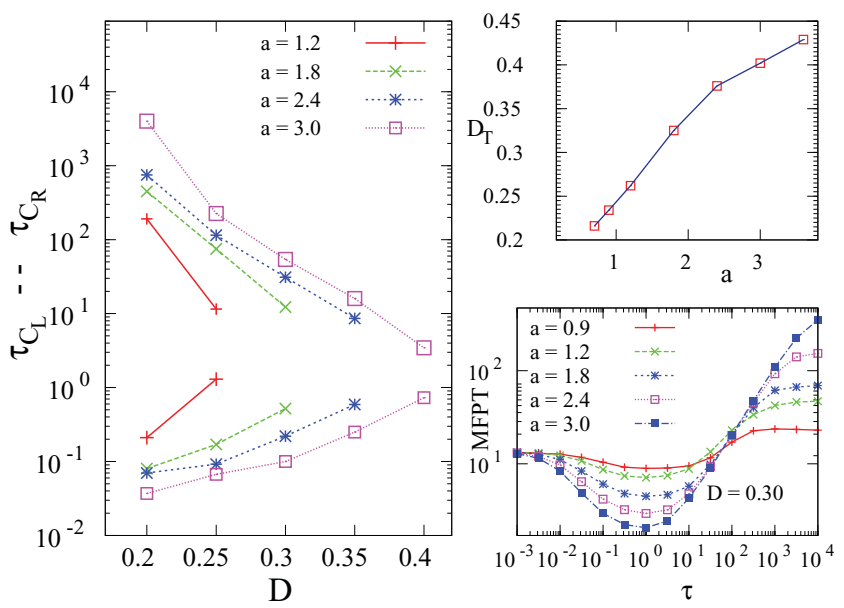

FIG. 6. (Color online) Left panel: Semilog plot of the values of MFPT at the two intersections in Fig. 2, $\tau_{C_{L}}$ and $\tau_{C_{R}}$ as a function of the noise intensity for different values of the amplitude of the dichotomous noise: $a=1.2,1.8,2.4,3.0$. Right top panel: Threshold value of the thermal noise intensity $D_{T}$ as a function of the amplitude $a$. Right bottom panel: MFPT as a function of the correlation time for different values of the amplitude $a$. All the other parameter values are as in Fig. 1 
square wave of amplitude $A_{r f}$. By increasing $A_{r f}$, the barrier height in the "down" configuration decreases, and the MFPT at resonance becomes lower as shown in Fig. 5(a) of Ref. [26] and in our Fig. 6.

The presence of a resonant behavior, as well as the crossing value at $\tau_{C_{L}}$, is also found in the behavior of the mean velocity of the Brownian particle. The left inset of Fig. 2 shows, in fact, this measure as a function of the correlation time of the fluctuating dichotomous force, calculated as $\bar{v}=N^{-1} \sum_{i=1}^{N} L / t_{i}$. For all the values of the asymmetry parameter, we see the presence, before the saturating behavior, of a weak maximum that corresponds to the resonant correlation time $\tau_{R}$. To check more carefully this maximum, we performed a set of simulations with different values of noise intensity, by increasing the sampling up to $N=120000$ realizations, for the asymmetry parameter value $k=0.25$. The results are shown in the inset of Fig. 5. We see that the maximum is more pronounced by increasing the noise intensity, while the curves show also a shift in the position of the maximum, according to the analogous shift in the minimum value of $\tau$. We can also see from Fig. 2 that for low values of the correlation times $\left(\tau<\tau_{C_{L}}\right)$ the mean velocity is higher for negative asymmetry and lower for positive one, while for $\left(\tau>\tau_{C_{L}}\right)$ it is the inverse. This feature gives rise to a current reversal in the ratchet, as predicted in other works [35-37] and whose occurrence has been also observed experimentally [38]. In fact, the difference between the mean velocities for positive asymmetry and for the negative one changes sign at $\tau=\tau_{C_{L}}$. If we consider an asymmetrical ratchet, this difference represents a net flux velocity, provided that the absence of any reflecting boundary in that case gives rise to changes in the values of the net velocity and on the correlation time where the reversal occurs. Both the presence of a maximum for $\tau \approx \tau_{R}$ and the crossing at $\tau \approx \tau_{C_{L}}$ are in total agreement with the behavior of the MFPT. This agreement fails for values of the correlation times higher than $\tau_{R}$.

While the MFPT curves increase in a different way and join at the second cross, the velocities decrease slightly, reaching a saturation value. This is because for high values of correlation time, the particle can easily cross the potential barrier when it is in its "down" configuration, reaching a relatively high speed because of the low crossing time. Conversely, when the potential is in the "up" configuration, the particle takes a longer time to cross the barrier, and so the contribution to the mean velocity becomes very small and relatively negligible. This means that, for high correlation times, $\bar{v}$ maintains a relatively high value because of the average between these two limit cases.

The above results, obtained for potential profiles with a single barrier, can be applied to the ratchet potential having the same asymmetric profile as elementary module. A set of calculations has been performed with the aim of joining the results obtained for the single-barrier case with the simplest ratchet case, i.e., a ratchet with two barriers only. Figure 7 shows the results in such a case, and the bottom right inset shows the corresponding elementary ratchet. The system consists of two asymmetric barriers without the presence of any reflecting boundary. The MFPT presents again a resonant correlation value $\tau_{R}$ that lies in the same region found in the single-barrier case. Now the MFPT is computed as the mean time spent by the Brownian particle to reach the position $x=1$ or $x=-1$, indifferently, starting at $x=0$. The particle follows, on average, the easiest path, and the MFPT represents

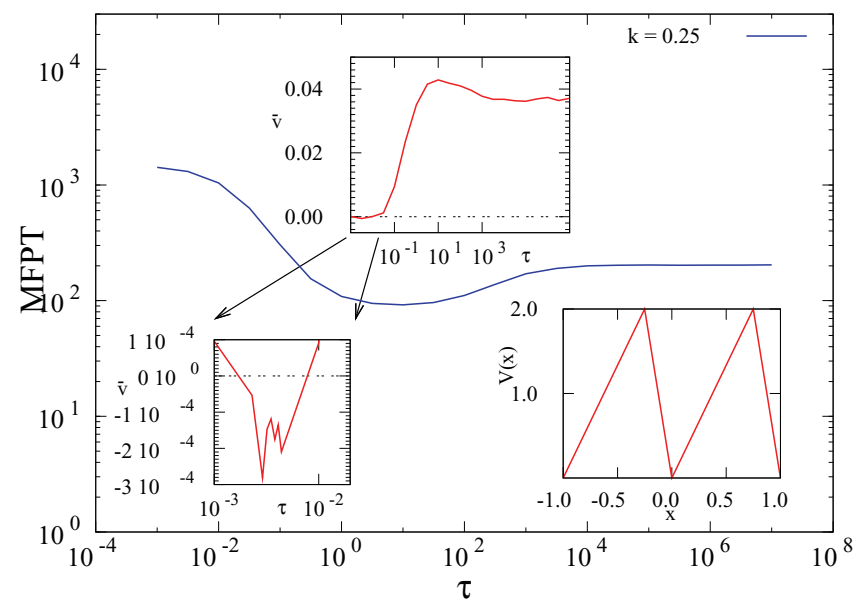

FIG. 7. (Color online) Log-log plot of MFPT as a function of the correlation time for the elementary ratchet composed by two single barriers only. The RA effect is observed. The MFPT is here the mean time taken by the Brownian particle starting at $x=0$ to reach the position $x=1$ or $x=-1$. The mean velocity of the particle is plotted in the upper inset. The mean velocity presents again a maximum at the same resonant value $\tau_{R}$. In the region of very low mean correlation time, the particle shows a very weak negative velocity (left inset), as a consequence of the different behavior of the mean velocities for the various asymmetries and the crossing of the curves shown in the inset of Fig. 2. The value of the noise intensity is $D=0.18$, and the amplitude of the dichotomous force is $a=1.2$. All the other parameters are as in Fig. 1.

here the minimum between the times spent to cross each single barrier. This means that the curve is lowered, and the RA effect is less pronounced. The mean velocity, plotted in the left inset of Fig. 7, shows again a maximum at the same resonant value $\tau_{R}$. For very low mean correlation times the mean velocity shows a very weak negative value (left bottom inset in Fig. 7). This means that a current reversal appears at a certain correlation time $\tau_{\text {rev }}$. This feature follows from the different behavior of the mean velocity in the two single asymmetric potential profiles (with $k=-0.25$ and $k=0.25$ ) reported in the left inset of Fig. 2, where the presence of the crossing value $\tau_{C_{L}}$ indicates a flux reversal as a function of $\tau$. The difference in value between $\tau_{\text {rev }}$ and $\tau_{C_{L}}$, as well as the difference in the value of the mean velocity of the Brownian particle, have to be ascribed to the presence of the reflecting boundary in the single-barrier case, which changes the traveling times of the particle and, so, the related mean velocities. The region of the lowest mean correlation time $(\tau \rightarrow 0)$ shows a zero current, in agreement with the detailed balance principle. In fact, that region represents the high-frequency fluctuation of the potential. In this condition the potential "felt" by the Brownian particle can be considered as a static one, and there is no net flux at the steady state.

As a last remark concerning the role of the potential shape on the the resonant activation effect, we report in Fig. 8 the results of calculations of the MFPTs by using symmetric smooth potentials. The corresponding static potentials used have the simple polynomial form

$$
V_{2 N}(x)=h 2^{2 N} \frac{x^{N}}{L^{N}}\left(1-\frac{x}{L}\right)^{N}
$$

with $h=2$ and $N=1,2,3$. 


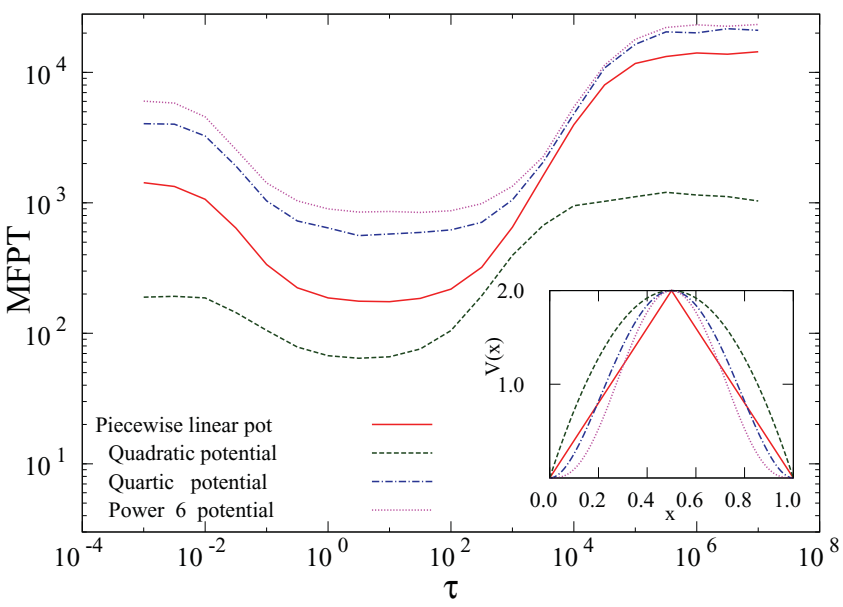

FIG. 8. (Color online) Log-log plot of MFPT as a function of the correlation time for different shape of symmetrical potential profiles. The RA is observed in all potential profiles. We note than for values of $\tau$ close to $\tau_{R}$ the logarithmic distances between the MFPT curves are approximatively constant. The parameter values are the same as those in Fig. 2.

As we can note in Fig. 8, the four curves of the MFPT differ from each other by about a constant quantity. This means that, in that region, their logarithmic distance is constant, and an exponential form factor has to be taken into account in order to estimate the MFPT for each potential shape.

\section{CONCLUSIONS}

By investigating the transitions from the initial well to the bottom of the adjacent potential well in different potential profiles, the asymmetry of the potential barrier arises naturally, and its role on the dynamics of the Brownian particle can be easily investigated. The presence of the asymmetry in the potential profiles together with the random fluctuating external force uniform in space gives rise to new features of the RA phenomenon. Two crossings of the MFPT curves with inversion of their relative behavior as a funcion of the correlation time of the dichotomous noise, is observed for low noise intensities $\left(D<D_{T}\right)$.

Moreover, we find a new peculiar crossing behavior in the activation process by varying the amplitude of the dichotomous noise. This theoretical result is in agreement with the experiment outcomes shown in Ref. [26].

The presence of a resonant behavior together with the crossing of the curves is also observed for the mean velocity of the Brownian particle as a function of $\tau$. This feature gives rise to a reversal current in the ratchet potential as observed in previous investigations [35-37] and, with a different model, also in Ref. [38].

The possibility of obtaining the experimental setup with the desired properties of asymmetry and periodicity of the potential structure [39], together with the crucial role of the asymmetry of the potential energy in governing the motion direction in nonequilibrium nanosystems [40], underlines the importance of further investigating the sign of the averaged directed velocity in ratchet potentials with the aim of obtaining kinematic control of Brownian motors.

\section{ACKNOWLEDGMENTS}

The authors wish to thank Martin Bier for useful and stimulating discussions. The authors acknowledge financial support from Spanish MICINN through Project No. FIS200801240, cofunded by FEDER funds, and the Italian Ministero dell'Istruzione, dell'Università e della Ricerca (MIUR).
[1] C. R. Doering and J. C. Gadoua, Phys. Rev. Lett. 69, 2318 (1992).

[2] M. Bier and R. D. Astumian, Phys. Rev. Lett. 71, 1649 (1993).

[3] P. Hänggi, Chem. Phys. 180, 157 (1994); P. Pechukas and P. Hänggi, Phys. Rev. Lett. 73, 2772 (1994).

[4] P. Reimann, Phys. Rev. Lett. 74, 4576 (1995).

[5] J. Iwaniszewski, Phys. Rev. E 54, 3173 (1996).

[6] M. Marchi, F. Marchesoni, L. Gammaitoni, E. Menichella Saetta, and S. Santucci, Phys. Rev. E 54, 3479 (1996); J. Iwaniszewski, I. K. Kaufman, P. V. E. McClintock, and A. J. McKane, ibid. 61, 1170 (2000).

[7] I. Klik and Y. D. Yao, Phys. Rev. E 57, 6180 (1998).

[8] M. Boguñá, J. M. Porra, J. Masoliver, and K. Lindenberg, Phys. Rev. E 57, 3990 (1998).

[9] A. L. Pankratov and M. Salerno, Phys. Lett. A 273, 162 (2000).

[10] B. Dybiec and E. Gudowska-Nowak, Phys. Rev. E 66, 026123 (2002).

[11] A. L. Pankratov and B. Spagnolo, Phys. Rev. Lett. 93, 177001 (2004).

[12] A. A. Dubkov, N. V. Agudov, and B. Spagnolo, Phys. Rev. E 69, 061103 (2004).
[13] E. V. Pankratova, A. V. Polovinkin, and B. Spagnolo, Phys. Lett. A 344, 43 (2005).

[14] A. Fiasconaro, B. Spagnolo, A. Ochab-Marcinek, and E. Gudowska-Nowak, Phys. Rev. E 74, 041904 (2006); A. Ochab-Marcinek, E. Gudowska-Nowak, A. Fiasconaro, and B. Spagnolo, Acta Phys. Pol. B 37, 1651 (2006).

[15] A. Fiasconaro, e-print arXiv:0805.2406 (15 May 2008).

[16] A. Wozinski and J. Iwaniszewski, Phys. Rev. E 80, 011129 (2009).

[17] R. N. Mantegna and B. Spagnolo, Phys. Rev. Lett. 84, 3025 (2000); J. Phys. IV 8, 247 (1998).

[18] Y. Yu and S. Han, Phys. Rev. Lett. 91, 127003 (2003).

[19] C. Schmitt, B. Dybiec, P. Hänggi, and C. Bechinger, Europhys. Lett. 74, 937 (2006).

[20] G. Sun, N. Dong, G. Mao, J. Chen, W. Xu, Z. Ji, L. Kang, P. Wu, Y. Yu, and D. Xing, Phys. Rev. E 75, 021107 (2007).

[21] C. Pan, X. Tan, Y. Yu, G. Sun, L. Kang, W. Xu, J. Chen, and P. Wu, Phys. Rev. E 79, 030104(R) (2009).

[22] V. N. Chizhevsky, Phys. Rev. E 80, 061139 (2009).

[23] Y. Fu, M. Zhu, and J. Xing, Phys. Biol. 7, 016013 (2010). 
[24] J. Li, Physica A 389, 7 (2010).

[25] J. Li and J. Łuczka, Phys. Rev. E 82, 041104 (2010).

[26] S. Miyamoto, K. Nishiguchi, Y. Ono, K. M. Itoh, and A. Fujiwara, Phys. Rev. B 82, 033303 (2010).

[27] R. N. Mantegna and B. Spagnolo, Phys. Rev. Lett. 76, 563 (1996).

[28] N. V. Agudov and B. Spagnolo, Phys. Rev. E 64, 035102(R) (2001); N. V. Agudov, ibid. 57, 2618 (1998).

[29] A. Fiasconaro, B. Spagnolo, and S. Boccaletti, Phys. Rev. E 72, 061110 (2005); A. Fiasconaro, D. Valenti, and B. Spagnolo, Physica A 325, 136 (2003).

[30] L. Gammaitoni, P. Hänggi, P. Jung, and F. Marchesoni, Rev. Mod. Phys. 70, 223 (1998); R. N. Mantegna, B. Spagnolo, and M. Trapanese, Phys. Rev. E 63, 011101 (2000).

[31] M. H. Devoret, J. M. Martinis, D. Esteve, and J. Clarke, Phys. Rev. Lett. 53, 1260 (1984).
[32] M. O. Magnasco, Phys. Rev. Lett. 71, 1477 (1993).

[33] T. Czernik, J. Kula, J. Łuczka, and P. Hänggi, Phys. Rev. E 55, 4057 (1997).

[34] J. Kula, T. Czernik, and J. Łuczka, Phys. Rev. Lett. 80, 1377 (1998).

[35] P. Reimann and T. C. Elston, Phys. Rev. Lett. 77, 5328 (1996).

[36] M. I. Dykman, H. Rabitz, V. N. Smelyanskiy, and B. E. Vugmeister, Phys. Rev. Lett. 79, 1178 (1997).

[37] D. G. Luchinsky, M. J. Greenall, and P. V. E. McClintock, Phys. Lett. A 273, 316 (2000).

[38] R. Gommers, P. Douglas, S. Bergamini, M. Goonasekera, P. H. Jones, and F. Renzoni, Phys. Rev. Lett. 94, 143001 (2005).

[39] L. Gorre-Talini, S. Jeanjean, and P. Silberzan, Phys. Rev. E 56, 2025 (1997).

[40] M. L. Dekhtyar, T. Ye. Korochkova, and V. M. Rozenbaum, Int. J. Quantum Chem. 110, 67 (2010). 